\title{
Minimally invasive parathyroidectomy without using intraoperative parathyroid hormone monitoring or gamma probe
}

Aykut Soyder ${ }^{1}$, Mustafa Ünübol ${ }^{2}$, İmran Kurt Ömürlü${ }^{3}$, Engin Güney², Serdar Özbaşs

ABSTRACT

'Department of General Surgery, Adnan Menderes University Faculty of Medicine, Aydın, Turkey ${ }^{2}$ Department of Endocrinology, Adnan Menderes University Faculty of Medicine, Aydın, Turkey ${ }^{3}$ Department of Biostatistics, Adnan Menderes University Faculty of Medicine, Aydın, Turkey ${ }^{4}$ Department of General Surgery, Güven Hospital, Ankara, Turkey

\section{Address for Correspondence} Aykut Soyder

Department of General Surgery, Adnan Menderes University Faculty of Medicine, Aydın, Turkey

Phone: +902564441256

e-mail:

asoyder@yahoo.com

Received: 12.12 .2013

Accepted: 28.06.2014

OCopyright 2015 by Turkish Surgical Association

Available online at www.ulusalcerrahidergisiorg
Objective: Minimal invasive parathyroidectomy (MIP) is a common surgical technique for the treatment of primary hyperparathyroidism (PHPT) and is usually done in conjunction with positive imaging techniques. We aimed to assess the results of this technique, performed without the use of intraoperative tests, in cases with PHPT caused by a single parathyroid adenoma.

Material and Methods: The data for patients who were diagnosed with PHPT were assessed retrospectively. Only those who had undergone a parathyroid adenoma localization study with ultrasonography (US) and parathyroid scintigraphy (PS) before the surgery, along with those patients for whom the MIP technique was routinely performed with frozen pathology, were included.

Results: The study group was made up of 65 patients who had undergone the MIP technique. The mean age of the patients was $56 \pm 14$ (20-81), with most being females [M/F: $19(29.2 \%) / 46(70.8 \%)]$. The mean calcium values before the operation were $11.24 \pm 1.26 \mathrm{mg} / \mathrm{dL}$ (8-15.5) (normal range: $8.4-10.2$ ), and the parathyroid hormone (PTH) values were $388 \mathrm{pg} / \mathrm{mL}$ (249-707.75). These same values, measured 24 hours after the operation, were determined as $9.04 \pm 1.04$ $\mathrm{mg} / \mathrm{dL}(6.8-13.9)$ and $27 \mathrm{pg} / \mathrm{mL}(6-86)$, respectively. The follow-up period for the patients was an average of $26.6 \pm 9.4$ (3-76) months, and only $3(4.6 \%)$ cases of persistent hyperparathyroidism were detected within this period.

Conclusion: Our success rate with MIP in PHPT cases was determined to be $95.4 \%$; therefore, this technique may be applied with a high success rate without any assistance from intraoperative tests, such as rapid serum PTH (rPTH) assays or gamma probes, in the presence of localization results of PS and US.

Key Words: Primary hyperparathyroidism, parathyroid adenoma, intraoperative, minimally invasive parathyroidectomy

\section{INTRODUCTION}

Primary hyperparathyroidism (PHPT) is a disease that develops as a result of excessive autonomous parathyroid hormone (PTH) secretion originating in the endocrine system and is a common cause of hypercalcemia. Approximately 1 out of every 500 women and 1 out of every 2000 men are affected by this disease annually (1). Since the start of serum calcium measurement in the 1970s, PHPT has become one of the most prevalent endocrine diseases (2). It occurs in 3 out of every 2000 people in Europe, and this ratio increases to 21 out of 1000 in the female population between the ages of 55-75 years (3). A single parathyroid adenoma is detected in $80 \%-85 \%$ of PHPT cases, parathyroid hyperplasia is detected in 15\%-20\%, multiple parathyroid adenomas are detected in 3\%, and carcinomas are detected in approximately $1 \%(4-6)$.

Medical centers with experience in parathyroid operations have recently begun to choose the surgical option rather than opt for long-term follow-up (7). In the past, the general surgical approach for patients with adenomas was to view all parathyroid glands by bilateral neck exploration and then remove the pathologic gland. However, the availability of ${ }^{99 \mathrm{~m} T c}$ sestamibi for parathyroid scintigraphy (PS) after 1992 has made unilateral exploration or selective parathyroidectomy applications more popular $(8,9)$. Selective parathyroidectomy may be open, minimally invasive, video-assisted, endoscopic, or radio-guided. As a result of these medical advances, the cause was identified as a single parathyroid gland in $85 \%$ of patients diagnosed with PHPT, and preoperative detection of the pathologic parathyroid gland can be performed with an accuracy rate of $95 \%-98 \%(10-12)$.

Patients and surgeons prefer the minimal invasive parathyroidectomy (MIP) technique over traditional exploration of the four parathyroid glands, owing to similar success rates, lower morbidity and recurrence ratios, and visible cost benefits. The measurement of rapid serum PTH (rPTH), performed just after the excision of the adenoma or radio-guided adenoma excision using a gamma probe during surgery, has increased the success rate of the MIP technique, for which this measurement is needed. When other studies compared the levels of PTH using the MIP techniques with the PTH levels before the excision, it was found that a decrease of more than $50 \%$ in the PTH level is an indicator of an operation's success. Once this is achieved, the surgery may be terminated. Different clinics have tried to attain the same achievement ratios with lower cost by using various combinations of these methods. 
In this study, we aimed to assess the results of the MIP technique, performed without using a gamma probe and with no rPTH measurement, in patients diagnosed with PHPT in our clinic.

\section{MATERIAL AND METHODS}

We retrospectively assessed the information for 98 patients who were diagnosed with PHPT owing to hypercalcemia and high PTH levels in our endocrinology clinic between January 2003 and December 2011. Only those patients who had met the criteria, determined by the NIH consensus panel between 2002 and 2008, who had undergone a parathyroidectomy, along with those who were symptomatic, were included in the study. Localization of the pathological gland(s) and thyroid tissue were evaluated in all patients preoperatively by both US and PS imaging of the neck. Double-phase images (early phase performed 15 minutes and late phase performed 2 hours after intravenous injection of $25 \mathrm{mCi}{ }^{99 \mathrm{mTC}}$ MIBI) were obtained and assessed. Focal activity retention continuing in the early and late images were accepted as being positive for parathyroid pathology scintigraphically. Normal serum calcium $(\mathrm{mg} / \mathrm{dL})$, PTH $(\mathrm{pg} / \mathrm{mL})$, postoperative (at 24 hours) calcium and PTH values, the weight of the removed parathyroid adenoma (g), and follow-up periods (months) of the cases were recorded. The histopathological diagnosis of patients who had a parathyroidectomy, the presence of the accompanying thyroid gland pathology, and the surgical procedure that was used and its complications were evaluated. The surgical procedure for patients diagnosed with PHPT was determined as either neck exploration or MIP according to the localization of the parathyroid adenomas and the presence of additional thyroid pathology. Surgery that was begun using the MIP technique was changed to classic neck exploration in cases in which it was difficult to locate the adenoma and in those patients with parathyroid hyperplasia. In the MIP technique, a cutaneous incision of 2.0-2.5 $\mathrm{cm}$ was performed at a predetermined localization according to the results of imaging methods in patients who were prepared under general anesthesia. The thyroid compartment was accessed by pulling the strap muscles apart after performing a minimal dissection in the subplatysmal zone. The pathological parathyroid gland was found and excised, and the tissue was removed and sent for frozen pathological examination (Figure 1, 2). The surgery was terminated after the tissue was identified as parathyroid tissue. Patients were discharged after the blood calcium and PTH measurements were collected at the postoperative $24^{\text {th }}$ hour. Tests for blood calcium and PTH were repeated on the third month after the operation. Persistent hyperparathyroidism was defined as hypercalcemia encountered within 6 months of the first operation. Recurrent hyperparathyroidism was defined as a return of elevated calcium and PTH levels after a 6-month period of eucalcemia. In our clinic, DEXA (dual-energy X-ray absorptiometry) is the preferred technique for measuring bone mineral density if patients' calcium and PTH levels are normal at 6 months postoperatively; and calcium, PTH, creatinine clearance, and serum albumin levels were measured annually. This study was approved by the appropriate ethics committee of Adnan Menderes University Faculty of Medi-

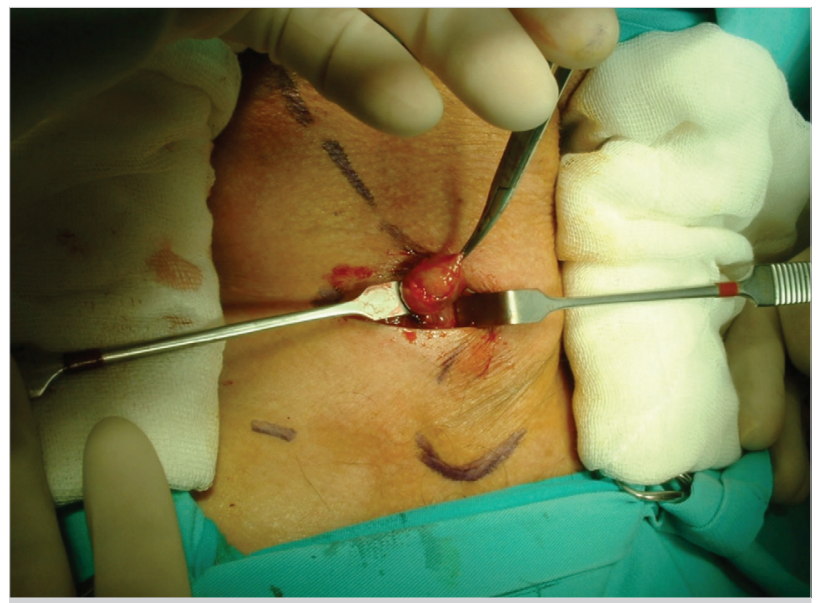

Figure 1. Image of right lower parathyroid adenoma after removal of sternocleidomastoid muscle to lateral and strap muscle to medial

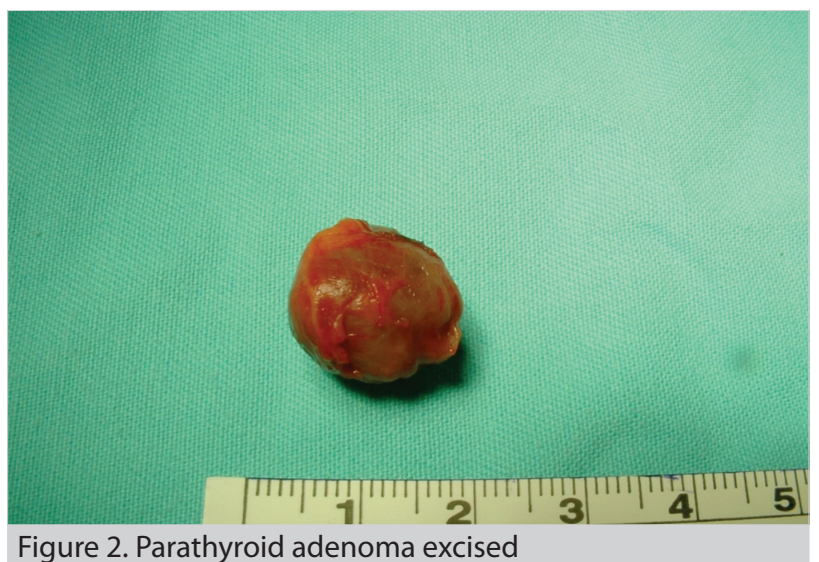

been performed in accordance with the ethical standards in the 1964 Declaration of Helsinki.

\section{Statistical Analysis}

The Statistical Package for the Social Sciences, version 17.0 software program (SPSS Inc., Chicago, IL, USA) was used for the statistical analysis. Conformity of quantitative data to normal distribution was analyzed with the Kolmogorov-Smirnov test. Age, pre- and postoperative serum calcium levels, PTH levels, the weight of the parathyroid adenoma tissue, and follow-up periods were recorded as mean \pm SD (minimum-maximum values) and median ( $25^{\text {th }}-75^{\text {th }}$ percentile), and gender distribution was given as a percentage. Correlation assessment between postoperative serum calcium and PTH values and the weight of the adenoma tissue was analyzed using Spearman's correlation test. The Mann-Whitney U-test was used in the evaluation of the weight of the adenoma tissue according to US and parathyroid gland scintigraphy. The Wilcoxon test was used to analyze the pre- and postoperative results of serum calcium and PTH. A value of $p<0.05$ was accepted as being statistically significant.

\section{RESULTS}

In our clinic, parathyroidectomy was performed in 98 patients diagnosed with PHPT. Neck exploration was done in 33 (33.6\%) patients, owing to parathyroid hyperplasia in 7 (7.1\%), 


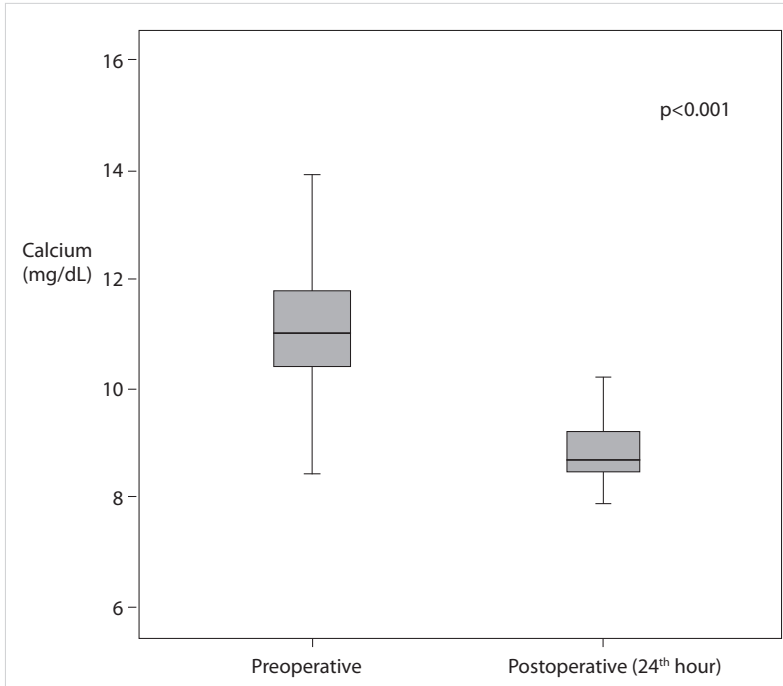

Figure 3. Calcium levels of patients measured before the surgery and at 24 hours

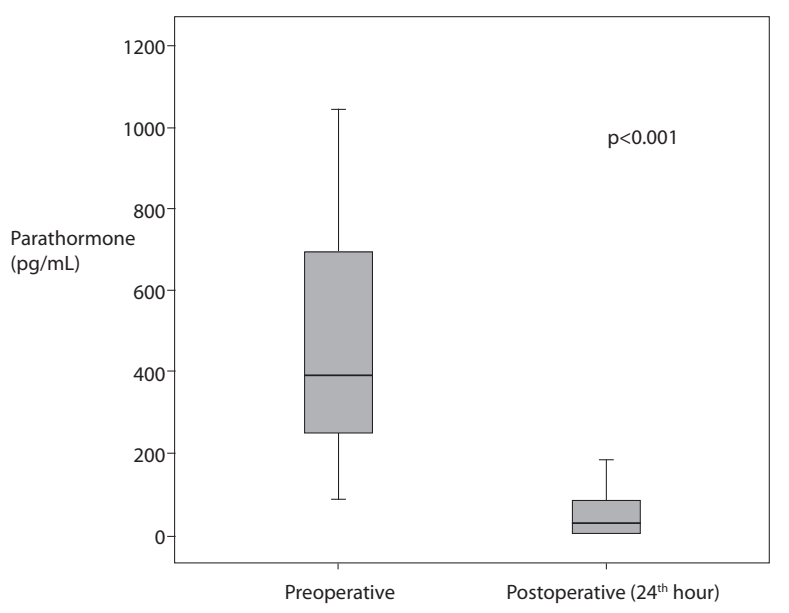

Figure 4. Parathormone levels of patients measured before the surgery and at 24 hours

suspicion of malignancy in $1(1 \%)$, and accompanying thyroid pathology presence in 25 (25.5\%) of the 98 patients. The MIP technique was completed successfully in the study group ( $n: 65)$. Complications were detected in 4 patients, including persistent hyperparathyroidism in $3(4.6 \%)$ cases in the study group and wound infection in $1(1 \%)$ case in the group that underwent neck exploration.

Our study group was composed of the 65 patients who underwent MIP. The mean age of these patients was $56 \pm 14$ $(20-81)$, and the majority was female [M/F: 19 (29.2\%)/46 $(70.8 \%)]$. The mean calcium values before the operation were 11.24 $\pm 1.26 \mathrm{mg} / \mathrm{dL}$ (8-15.5), and the median PTH values were $388 \mathrm{pg} / \mathrm{mL}$ (249-707.75). All specimens were reported as parathyroid adenoma as a result of a definitive pathology report. The mean calcium values and median PTH values measured at the $24^{\text {th }}$ postoperative hour were determined as $9.04 \pm 1.04 \mathrm{mg} / \mathrm{dL}$ (6.8-13.9) and $27 \mathrm{pg} / \mathrm{mL}$ (6-86), respectively. The mean weight of the removed parathyroid glands was $1.15 \mathrm{~g}$ (0.7-1.8) (Table 1). When the mean calcium and PTH values of the patients both before and after the opera-
Table 1. Clinical characteristics and laboratory values of patients

\begin{tabular}{|lc|}
\hline Patient Information & $\mathrm{n}: \%$ \\
\hline Total number & 65 \\
\hline Age & $56 \pm 14$ \\
\hline Gender (M/F) & $19(29.2 \%) / 46(70.8 \%)$ \\
\hline Preoperative calcium values (mg/dL)* & $11.24 \pm 1.26$ \\
\hline Preoperative PTH (pg/mL)* & $388(249-707.75)$ \\
\hline Postoperative calcium values (mg/dL) & $9.04 \pm 1.04$ \\
\hline Postoperative PTH (pg/mL) & $27(6-86)$ \\
\hline Adenoma weight (g) & $1.15(0.7-1.8)$ \\
\hline Average follow-up period (month) & $26.6 \pm 9.4$ \\
\hline Surgical procedure applied & \\
\hline & MIP
\end{tabular}

\section{Surgical complication Persistent hyperparathyroidism $3(4.6 \%)$}

*Normal range: Calcium 8.4-10.2 mg/dL, PTH 16-88.3 pg/mL MIP: minimally invasive parathyroidectomy; PTH: parathormone

tion were compared, a statistically significant decrease was observed $(p<0.05)$ (Figures 3, 4).

When the results of the imaging methods performed before the surgery were evaluated, the positive predictive values of US and PS, individually and in combination, were $80.6 \%, 91.8 \%$, and $98.9 \%$, respectively. No statistically significant relationship was detected between the weight of the adenoma removed and serum calcium and PTH values of the patients (Table 2), whereas a statistically significant relationship was found between the weight of the adenoma and the ratios for the ability to locate the adenoma by US and PS $(p<0.05)$ (Table 3$)$. The average follow-up period for the patients was 26.6 \pm 9.4 (3-76) months, and persistent hyperparathyroidism was detected in $3(4.6 \%)$ cases within this period. In the follow-up process of 62 patients, calcium and PTH levels were found to be within normal limits.

\section{DISCUSSION}

The use of less invasive procedures has become prominent with the presence of better localization techniques in the surgical treatment of PHPT. The MIP technique performed for PHPT has been accepted by experienced medical centers as a safe and lowcost technique with the same success rates (>95\%) as bilateral neck exploration. When we reviewed the studies performed in our country, it was discovered that there have been only 5 studies reporting more than 100 cases, and the number of cases for patients between 2.5 and 15 years old varies between 4 and 36 (13). Reaching a case count of 100 within 8 years at our clinic, with $50 \%$ of these cases occurring in the last 3 to 4 years, is an important accomplishment. Furthermore, it has been noted that MIP was not prominent in any of the previous studies (14-16). Application ratios of $66.3 \%$ for PHPT treatment in our clinic have been observed, and this ratio has increased to $78.5 \%$ within the last 5 years. The goal in the surgical treatment of PHPT is to remove 


\section{Table 2. Results of correlation between adenoma weight} and calcium and PTH

\begin{tabular}{|lcc|}
\hline & \multicolumn{2}{c|}{ Adenoma weight } \\
\hline Parameters & $\mathbf{r}$ & $\mathbf{p}$ \\
\hline Serum calcium & -0.114 & 0.283 \\
\hline Serum PTH & -0.091 & 0.396 \\
\hline PTH: parathormone & & \\
\hline
\end{tabular}

Table 3. Statistically significant results between parameters

Adenoma weight

$\begin{array}{lcc}\text { Parameters } & \text { Median }(25-75 \text { percentile) } & \text { p } \\ \text { USG } & & \\ \text { Present }(n=6) & 0.30(0.28-0.45) & <0.001 \\ \text { Absent }(n=84) & 1.30(0.80-1.95) & \\ \text { Scintigraphy } & & <0.001 \\ \text { Present }(n=16) & 0.65(0.53-0.88) & \\ \text { Absent }(n=74) & 1.40(0.80-2.00) & \\ \text { USG: ultrasonography } & & \end{array}$

the pathological parathyroid gland and to provide a normocalcemic level after the surgery by leaving normal parathyroid glands intact (17). Bilateral neck operations, first performed in 1925, have become a standard treatment for PHPT with a successful parathyroidectomy. The MIP technique, which is devoted to searching for the parathyroid adenoma in a narrow zone and removing the adenoma, done in conjunction with recent preoperative imaging techniques, combines a high success rate with low cost, along with similar success rates, less postoperative pain, better patient compliance, and fewer hypocalcemic complications $(18,19)$.

The primary field of discussion for parathyroid surgery is adenomas, the source of more than $80 \%$ of PHPTs. However, discussions have also continued to center around factors, such as the effective localization of adenomas, imaging methods, selection of the correct anesthesia for surgery, surgical approaches, indications for surgery in asymptomatic PHPT, use of the rPTH test during surgery, and use of the gamma probe. Studies have found that for $85 \%-90 \%$ of cases diagnosed with PHPT with a single adenoma, surgery has been successful with single gland resection (20). The success of the MIP technique depends on the ability to locate the parathyroid adenoma before the surgery. Both US and PS have been found to be effective imaging methods for the localization of the adenoma and have been recommended as the primary methods to be used (21). The sensitivity for detection of a parathyroid adenoma with US is $70 \%-80 \%$. However, with regard to the success of US for the detection of parathyroid glands that are slightly larger than normal, there is a large difference in published studies regarding its efficacy (11). It has been reported that adenomas detected in our country have a larger size; therefore, this feature increases the success of US (22-25).
Another reason for using US is that thyroid disease occurs in $20 \%-75 \%$ of patients with PHPT, particularly in endemic regions. This type of imaging allows for the detection of accompanying thyroid diseases and for the evaluation of present thyroid nodules for malignancy. The positive predictive value of US alone in the localization of single adenomas was $80.6 \%$ in our study, and thyroidectomy was added to parathyroidectomy at our clinic because of the accompanying thyroid pathology detected in 25 patients. Furthermore, according to the results of our study, the relationship between the localization success of US and the weight of the adenoma was statistically significant $(p<0.001)$. PS has been used as a key component in locating the adenoma in many studies, and it has been reported to be more successful than US. The sensitivity and specificity of PS regarding the detection of adenomas were $91 \%$ and $98.8 \%$, respectively. The sensitivity for the detection of parathyroid adenomas using a combination of US and PS increased to $95 \%$ or more (26). In our study, the positive predictive value of PS alone to detect parathyroid adenomas was $91.8 \%$, and the ratio increased to $98.9 \%$ when it was combined with US.

However, some medical centers use the gamma probe and rPTH measurement methods individually or in combination to increase the achievement ratios of the MIP technique. Surgery is accepted as being successful if there is more than a $50 \%$ decrease in PTH level when an rPTH measurement is performed just before the surgery and again 15 minutes after the removal of the adenoma. Achievement rates of $95 \%-100 \%$ have been reported with the use of $\mathrm{rPTH}$ and the MIP technique (27-31). However, some studies have indicated that using the rPTH measurement is not necessary due to the high cost, difficulty in the standardization of the sampling time, prolonged operation period, and false negative and positive ratios (32-34). In other studies, high achievement rates of $95 \%-98 \%$ in the MIP technique have been reported without using $\mathrm{rPTH}(35,36)$. It has also been shown in 2 studies that failure in MIP surgery with the use of rPTH could not be prevented $(37,38)$.

Measurements of rPTH and the use of the gamma probe may be useful in persistent cases, in situations in which there is nonconformity of PS and US in localization studies, and in cases with a second adenoma (37).

Three (4.6\%) patients, determined to have persistent hyperparathyroidism, did not accept a re-operation because of comorbid conditions (advanced age, diabetes, and hypertension), resulting in a lack of data in the presence of a secondary adenoma.

\section{CONCLUSION}

The MIP technique, performed in patients with PHPT, is an effective treatment option with low morbidity. The success rate that we obtained without using additional methods during surgery performed after localization studies using US and PS imaging, such as the gamma probe and $\mathrm{rPTH}$, was $95.4 \%$. We believe that the most important reason for the high success rate in parathyroid surgery is the requirement of multidisciplinary studies in the diagnosis phase, 
along with the localization studies performed before the surgery.

Ethics Committee Approval: Ethics committee approval was received for this study from the ethics committee of Adnan Menderes University Faculty of Medicine (B.30.2.ADÜ.0.20.05.00/050.040-120).

Informed Consent: Due to the retrospective design of the study, the informed consent was not obtained.

Peer-review: Externally peer-reviewed.

Author Contributions: Concept - A.S., M.Ü.; Design - A.S., M.Ü., S.Ö., E.G.; Supervision - S.Ö., E.G.; Funding - A.S., M.Ü.; Materials - A.S., M.Ü.; Data Collection and/or Processing - A.S., M.Ü.; Analysis and/or Interpretation - İ.K.Ö., S.Ö., E.G.; Literature Review - A.S., M.Ü.; Writer - A.S., M.Ü.; Critical Review - S.Ö., E.G., I.K.Ö.

Conflict of Interest: No conflict of interest was declared by the authors.

Financial Disclosure: The authors declared that this study has received no financial support.

\section{REFERENCES}

1. Bilezikian JP, Silverberg SJ. Clinical spectrum of primary hyperparathyroidism. Rev Endocr Metab Disord 2000; 1: 237-245.

2. Sywak MS, Robinson BG, Clifton-Bligh P, Reeve TS, Barraclough $\mathrm{BH}$, Fick $\mathrm{GH}$, et al. Increase in presentations and procedure rates for hyperparathyroidism in Northern Sydney and New SouthWales. Med J Aust 2002; 177: 246-249.

3. Adami S, Marcocci C, Gatti D. Epidemiology of primary hyperparathyroidism in Europe. J Bone Miner Res 2002; 17: 18-23.

4. Fraser WD. Hyperparathyroidism. Lancet 2009; 374: 145-158.

5. Marcocci C, Cetani F, Rubin MR, Silverberg SJ, Pinchera A, Bilezikian JP. Parathyroid carcinoma. J Bone Miner Res 2008; 23: 1869-1880.

6. Bilezikian JP, Potts JT Jr, Fuleihan Gel-H, Kleerekoper M, Neer R, Peacock $M$, et al. Summary statement from a workshop on asymptomatic primary hyperparathyroidism: a perspective for the $21^{\text {st }}$ century. J Bone Miner Res 2002; 17 (Suppl 2): N2-11.

7. Coker LH, Rorie K, Cantley L, Kirkland K, Stump D, Burbank N, et al. Primary hyperparathyroidism, cognition and health-related quality of life. Ann Surg 2005; 242: 642-650.

8. Grant CS, Thompson G, Farley D, van Heerden J. Primary hyperparathyroidism surgical management since the introduction of minimally invasive parathyroidectomy: Mayo Clinic experience. Arch Surg 2005; 140: 472-478.

9. Sosa JA, Udelsman R. Minimally invasive parathyroidectomy. Surg Oncol 2003; 12: 125-134.

10. Haber RS, Kim CK, Inabnet WB. Ultrasonography for preoperative localization of enlarged parathyroid glands in primary hyperparathyroidism: comparison with $99 \mathrm{~m}$ technetium sestamibi scintigraphy. Clin Endocrinol 2002; 57: 241-249.

11. Geatti O, Shapiro B, Orsolon PG, Proto G, Guerra UP, Antonucci F, et al. Localization of parathyroid enlargement: experience with technetium 99m methoxyisobutylisonitrile and thallium-201 scintigraphy, ultrasound and computed tomography. Eur J Nucl Med 1994; 21: 17-23.

12. Casara D, Rubello D, Pelizzo MR, Shapiro B. Clinical role of $99 \mathrm{mTCO} 4 \mathrm{MIBI}$ scan, ultrasound and intra-operative gamma probe in the performance of unilateral and minimally invasive surgery in primary hyperparathyroidism. Eur J Nucl Med 2001; 28: 1351-1359.

13. Çaycı M, Karahan Ö, Eryılmaz MA, Sevinç B, Arslan K, Okuş A, et al. Factors affecting the development of the parathyroid surgery. Ulusal Cer Derg 2011; 27: 98-102.
14. Erbil Y, Barbaros U, Yanik BT. Impact of gland morphology and concomitant thyroid nodules on preoperative localization of parathyroid adenomas. Laryngoscope 2006; 116: 580-585.

15. Erbil Y, Barbaros U, Tukenmez M, Işsever H, Salmaslioğlu A, Adalet l, et al. Impact of adenoma weight and ectopic location of parathyroid adenoma on localization study results. World J Surg 2008; 32: 566-571.

16. Kotan Ç, Sümer A, Öztürk M, Algün E, Ayakta H, Kösem M, et al. Primary hyperparathyroidism: Experience with 149 cases in Van Region. Journal of Dialogue in Endocrinology 2008; 2: 27-32.

17. Palazzo F, Sadler GP. Minimally invasive parathyroidectomy, heralds a new era in the treatment of primary hyperparathyroidism. BMJ 2004; 328: 849-850.

18. Goldstein RE, Blevins L, Delbeke D, Martin WH. Effect of minimally invasive radioguided parathyroidectomy on efficacy, length of stay, and costs in the management of primary hyperparathyroidism. Ann Surg 2000; 231: 732-742.

19. Udelsman R. Six hundred fifty-six consecutive explorations for primary hyperparathyroidism. Ann Surg 2002; 235: 665-670.

20. Mariani G, Gülec SA, Rubello D. Preoperative localization and radioguided parathyroid surgery. J Nucl Med 2003; 44: 1443-1458.

21. Korukluoğlu B, Kıyak G, Çelik A, Ucar AE, Ergül E, Kuşdemir A. Our surgical approach to parathyroid adenoma and the role of localization studies. Türkiye Klinikleri J Med Sci 2008; 28: 24-29.

22. Mihai R, Palazzo FF, Gleeson FV, Sadler GP. Minimally invasive parathyroidectomy without intraoperative parathyroid hormone monitoring in patients with primary hyperparathyroidism. $\mathrm{Br} J$ Surg 2007; 94: 42-47.

23. Gil-Cárdenas A, Gamino R, Reza A, Pantoja JP, Herrera MF. Is intraoperative parathyroid hormone assay mandatory for the success of targeted parathyroidectomy? J Am Coll Surg 2007; 204: 286-290.

24. Kirdak T, Duh QY, Kebebew E, Clark OH. Do patients undergoing parathyroidectomy for primary hyperparathyroidism in San Francisco; CA, and Bursa, Turkey, differ? Am J Surg 2009; 198: 188-192.

25. Berber E, Parikh RT, Ballem N, Garner CN, Milas M, Siperstein AE. Factors contributing to negative parathyroid localization: An analysis of 1000 patients. Surgery 2008; 144: 747-749.

26. Denham DW, Norman J. Cost-effectiveness of preoperative sestamibi scan for primary hyperparathyroidism is dependent solely upon the surgeon's choice of operative procedure. J Am Coll Surg 1999; 186: 293-305.

27. Sackett WR, Barraclough B, Reeve TS, Delbridge LW. Worldwide trends in the surgical treatment of primary hyperparathyroidism in the era of minimally invasive parathyroidectomy. Arch Surg 2002; 137: 1055-1059.

28. Vignali E, Picone A, Materazzi G, Steffe S, Berti P, Cianferotti L, et al. A quick intraoperative parathyroid hormone assay in the surgical management of patients with primary hyperparathyroidism: a study of 206 consecutive cases. Eur J Endocrinol 2002; 146: 783788.

29. Proctor MD, Sofferman RA. Intraoperative parathyroid hormone testing: what have we learned? Laryngoscope 2003; 113: 706-714.

30. Burkey SH, Van Heerden JA, Farley DR, Thompson GB, Grant CS, Curlee KJ. Will directed parathyroidectomy utilizing the gamma probe or intraoperative parathyroid hormone assay replace bilateral cervical exploration as the preferred operation for primary hyperparathyroidism? World J Surg 2002; 26: 914-920.

31. Irvin GL $3^{\text {rd }}$, Solorzano CC, Carneiro DM. Quick intraoperative parathormone assay: Surgical adjunct to allow limited parathyroidectomy, improve success rate, and predict outcome. World J Surg 2004; 28: 1287-1292.

32. Weber CJ, Ritchie JC. Retrospective analysis of sequential changes in serum intact parathyroid hormone levels during conventional parathyroid exploration. Surgery 1999; 126: 1139-1143. 
33. Lee JA, Inabnet WB $3^{\text {rd }}$. The Surgeons Armamentarium to the surgical treatment of primary hyperparathyroidism. J Surg Oncol 2005; 89: 130-135.

34. Stalberg P, Sidhu S, Sywak M, Robinson B, Wilkinson M, Delbridge L. Intraoperative parathyroid hormone measurement during minimaly invasive parathyroidectomy: Does it "Value-add" to decision-making? J Am Coll Surg 2006; 203: 1-6.

35. Shabtai M, Ben-Haim M, Muntz Y, Vered I, Rosin D, Kuriansky J, et al. 140 consecutive cases of minimally invasive, radio-guided parathyroidectomy: lessons learned and long-term results. Surg Endosc 2003; 17: 688-691.
36. Ollila DW, Caudle AS, Cance WG, Kim HJ, Cusack JC, Swasey JE, et al. Successful minimally invasive parathyroidectomy for primary hyperparathyroidism without using intraoperative parathyroid hormone assays. Am J Surg 2006; 191: 52-56.

37. Garner SC, Leight GS Jr. Initial experience with intraoperative PTH determinations in the surgical management of 130 consecutive cases of primary hyperparathyroidism. Surgery 1999; 126: 1132-1137.

38. Gordon LL, Snyder WH $3^{\text {rd }}$, Wians F Jr, Nwariaku F, Kim LT. The validity of quick intraoperative parathyroid hormone assay: an evaluation in seventy-two patients based on gross morphologic criteria. Surgery 1999; 126: 1030-1035. 